\title{
PSYCHOLOGY
}

\section{Risks, real and imagined}

\author{
Older adults are at high risk of suffering debilitating health effects from COVID-19. Effective communication of \\ associated risks is therefore paramount. A new study finds that imagining a personalized disease transmission \\ event amplifies perceived risk and bolsters risk-related information seeking in older age.
}

\section{Adam Bulley and Daniel L. Schacter}

Y ou've invited four of your closest friends and family members over for dinner. One of your guests starts coughing during the main course. Three days later, they have tested positive for COVID-19 and shortly thereafter you develop symptoms yourself. Tragically, scenarios like this one have played out countless times over the past 18 months of the coronavirus pandemic. In this issue of Nature Aging, Sinclair and colleagues ${ }^{1}$ show that a guided imagination exercise involving simply playing out such a scenario in the mind's eye can have wide-reaching effects on how older adults grapple with their risk of contracting the disease, with broad implications for communicating public health information.

Given that increased age is associated with an elevated risk of hospitalization, intensive care unit admission and death due to COVID-19, Sinclair et al. set out to tailor a risk-related intervention to the needs of older adults. Effectively communicating risk information is always difficult, but tailoring communication for older adults is more daunting still for at least two reasons. First, older adults are particularly susceptible to believing and sharing misinformation online ${ }^{2}$. Second, while most people exhibit somewhat of an optimism bias, believing they are less likely to suffer a heart attack or robbery than average, older adults are particularly likely to see the world through rose-tinted glasses. One possible reason for the age-related optimism bias is that older adults are less likely to change their beliefs when confronted with undesirable information ${ }^{3}$.

In the Sinclair et al. study, 546 participants from the USA, ranging in age from 18 years to 81 years old, completed an online survey where they were asked to rate the risk of engaging in 15 different activities during the pandemic, from picking up takeout to attending a party (Fig. 1). They completed these ratings three times: once early in the study, again after either a personalized imagination intervention or a control manipulation, a

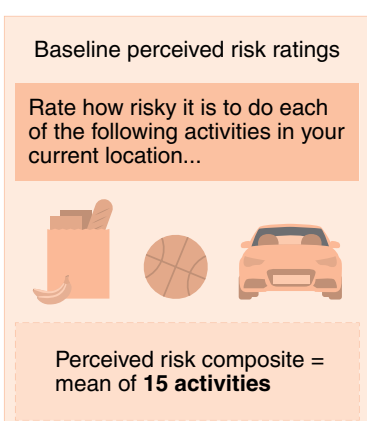

d

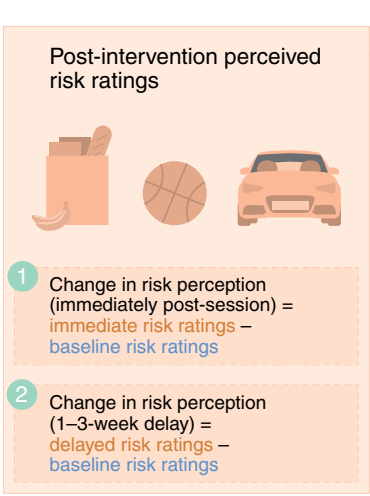

c

Numerical risk estimation and feedback

Think about a hypothetical event in your location..

Estimate the probability that in a group of $\mathbf{2 5}$ people, someone is infected

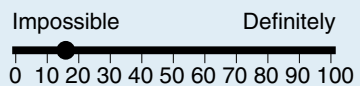

Prediction error $=$ mean (actual risk - estimated risk)
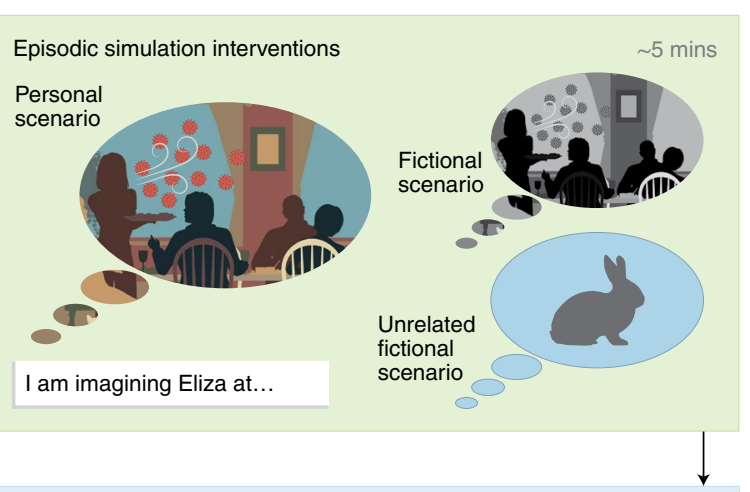

Fig. 1 | Harnessing the imagination to communicate risk. a, Sinclair et al. asked participants to rate the risk of 15 everyday activities, such as grocery shopping, playing sports or taking a taxi, on a scale from 1 (low risk) to 5 (high risk). The authors created a 'perceived risk' score from participants' average ratings. b. In the episodic simulation intervention, participants were randomly assigned to one of three guided imagination exercises: in the experimental condition, they imagined a personal COVID-19 transmission event, while in the control conditions, they imagined a fictional character experiencing the same event or an unrelated fictional scenario about rabbits falling ill. Schematic adapted with permission from Robert Adrian Hillman / Alamy Stock Photo. c, Following the simulation intervention, participants estimated the probability that at least one person was infected with COVID-19 at local events of different sizes (5, 10, $25,50,100,250$ or 500 people) and received feedback based on actual risk prevalence data from their county. The experimenters calculated 'prediction errors' as the average discrepancy between actual risk and estimated risk. d, Participants rated the risk of everyday activities twice more after the intervention, allowing the experimenters to calculate changes in risk perception immediately after the intervention, and again after a 1-3-week delay. Relative to the control conditions, personalized episodic simulation amplified risk perception to a greater extent in older adults than younger adults.

and once more between 1 and 3 weeks after the intervention. During the personalized intervention, participants vividly pictured a COVID-19 disease transmission event involving the hospitalization of a loved one and their own infection. They selected four people they might invite over for dinner, such as 'my sister Eliza', before working through a series of prompts for generating vivid mental imagery, such as "close your 
eyes and try to visualize what their faces look like". Throughout, participants typed short blurbs describing what they imagined and how the events would make them feel. Participants in two control groups imagined either an impersonal, fictional version of this scenario or an unrelated scenario.

After these manipulations, participants played a game where they estimated the purely numerical risk that at least one person would be infected with COVID-19 in variously sized gatherings, and received feedback based on the actual case prevalence where they lived. The authors were able to use this game to calculate how readily participants updated their perception of everyday risk based on the mismatch between their numerical estimates and actual local risk levels.

At the 1-to-3-week follow-up, older adults (that is, adults aged $60-81$ years) who underwent the personalized imagination intervention perceived greater risk in everyday activities compared with older adults in the control conditions and with younger adults. Such an effect is potentially surprising considering that older adults exhibit deficits in imagining past and future scenarios ${ }^{4}$, and episodic simulation interventions in other domains such as decision-making have been found less effective for older adults ${ }^{5}$. Recent work also finds no effect of episodic simulation on perceived risks in the context of other threats like terrorism ${ }^{6}$. Sinclair et al. point out that their personalized imagination intervention taps directly into the cognitive profile of older adults by evoking a personally relevant social event occurring with loved ones. Moreover, the imagination intervention was paired here with the numerical risk game that provided real statistical feedback. Though the design of the current study makes it hard to discern because it lacks a control condition involving only the imagination manipulation, it's possible that intervention effects resulted from some unique interaction between the imagination manipulation and the risk estimation feedback.

In the risk estimation game, older adults were worse than younger adults at using feedback to update their beliefs about everyday risk. This finding accords with previous research showing that older age is associated with difficulties learning from errors ${ }^{7}$. Sinclair et al. do not report whether older adults updated their beliefs differentially when actual virus rates were better versus worse than participants expected, but one possibility is that older adults are more resistant to bad news about COVID-19 transmission, mirroring their optimism elsewhere ${ }^{3}$. It remains to be seen if this bias would also be amenable to modification. One promising finding is that after receiving the personalized imagination intervention, older adults in this condition reported consuming more information about local COVID-19 risk levels, which might have played a role in amplifying their perception of risk. This could also be one reason why the intervention affected risk perception differently in older adults only after the 1-3-week delay.

The Sinclair et al. study is the latest in a surge of research into applying the science of episodic simulation - the cognitive processes involved in thinking about or imagining specific personal events. Previous research has identified a range of everyday functions that episodic simulation supports, such as emotion regulation, facilitating prospective memory, problem-solving and goal pursuit ${ }^{8,9}$. The current work adds 'bolstering disease risk perception' to the list of possible applications.

One reason episodic simulation interventions might be so effective across diverse domains is that they interact with common biases and heuristics. For instance, despite general optimism, people tend to overestimate how negative an event will make them feel, partly because they zero-in on its worst elements rather than taking a broader view of the context in which it will occur ${ }^{10}$. An imagination intervention might boost this bias by encouraging the vivid simulation of gritty details and the neglect of contextual factors. The availability heuristic, whereby people predict the likelihood of events based on how easily they are called to mind ${ }^{11}$, might be similarly boosted because imagination brings threatening possibilities vividly to the forefront of attention. Indeed, other research shows that repeatedly imagining emotional events leads people to estimate them as more likely or plausible ${ }^{12}$.

What, then, of optimism itself? The Sinclair et al. findings might be useful for tuning down the optimism bias in older adults. The authors argue that instilling such risk-averse attitudes offers clear benefits to public health given the perils of contracting COVID-19 in older adulthood. Maybe so. It's important to note, however, that the increase in information seeking elicited by the imagination intervention did not improve the actual accuracy of risk estimation, meaning that older adults in the study may have become more risk averse, regardless of real risk.

As with any new intervention, we will need a sober look at any possible side effects. For instance, the current data from Sinclair et al. do not allow us to determine the breadth of the imagination intervention effect. If the manipulation works by increasing stress or general anxiety, rather than disease-specific risk perception, the intervention might generate paradoxical effects such as increasing vaccine hesitancy rather than reducing it, or deepening the gulf of loneliness for older individuals already profoundly fearful of the disease-transmission dangers of social interaction.

The ability to travel mentally in time, to visit possible dangers ahead of their occurrence, is one of the foundational adaptive capacities of our species. Nonetheless, this same adaptive process is also central to anxiety disorders and other psychopathologies ${ }^{13}$. Optimism may be one protective balm against these afflictions, and we should be wary of dispensing with it. After all, optimism has long been associated with benefits to physical health and psychological wellbeing ${ }^{14}$.

Like any new finding, this study needs careful replication. Ideally, follow-up experiments will be conducted in person rather than online, under well-controlled settings where experimenters can both isolate specific mechanisms and establish the generalizability of any effects. The door is now open for new questions. Do imagination effects on risk perception in older adults extend to actual behaviours? At a neural level, are intervention effects associated with activation in the core network of brain regions previously implicated in mentally simulating past and future events ${ }^{9}$ ? Would this intervention have been more effective with more vivid or emotionally significant mental imagery? Could such an intervention be made more powerful still by using virtual reality to present older adults with a rendering of the scenario they imagined?

And what about the other side of the coin? Recent research shows that a similar guided imagination intervention involving mentally simulating a positive travel experience can actually reduce the perceived risk of contracting COVID-19 in a mixed-age cohort ${ }^{15}$. This finding implies that anyone attempting to harness the imagination for public health communication should be highly circumspect about what exactly consumers use their imaginations to conjure up.

Sinclair et al. present a promising extension of episodic simulation research into the domain of real-world risk perception. Further research on how both younger and older adults envisage the consequences of their actions might help in addressing other large-scale collective 
threats, offering hope for a productive applied science of the imagination.

\section{Adam Bulley ${ }^{10}{ }^{1,2}$ and}

Daniel L. Schacter (ID)

${ }^{1}$ Department of Psychology, Harvard University, Cambridge, MA, USA. ${ }^{2}$ The University of Sydney, School of Psychology and Brain and Mind Centre, Sydney, New South Wales, Australia.

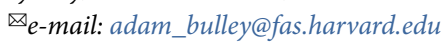

Published online: 5 August 2021

https://doi.org/10.1038/s43587-021-00097-5

References

1. Sinclair, A. H. et al. Nat. Aging https://doi.org/10.1038/s43587021-00095-7 (2021).
2. Brashier, N. M. \& Schacter, D. L. Curr. Dir. Psychol. Sci. 29 , 316-323 (2020).

3. Chowdhury, R., Sharot, T., Wolfe, T., Düzel, E. \& Dolan, R. J. Psychol. Med. 44, 2003-2012 (2014).

4. Schacter, D. L., Devitt, A. L. \& Addis, D. R. in The Oxford Encyclopedia of Psychology and Aging (Ed. Knight, B. G.) 1-22 (Oxford Univ. Press, 2018).

5. Sasse, L. K., Peters, J. \& Brassen, S. Front. Aging Neurosci. 9 , 58 (2017).

6. Bø, S. \& Wolff, K. Front. Psychol. 10, 2333 (2019).

7. Samanez-Larkin, G. R., Worthy, D. A., Mata, R., McClure, S. M. \& Knutson, B. Cogn. Affect. Behav. Neurosci. 14, 672-682 (2014).

8. Bulley, A., Redshaw, J. \& Suddendorf, T. in The Cambridge Handbook of the Imagination (Ed. Abraham, A.) 425-444 (Cambridge Univ. Press, 2020)

9. Schacter, D. L., Benoit, R. G. \& Szpunar, K. K. Curr. Opin. Behav. Sci. 17, 41-50 (2017)

10. Gilbert, D. T. \& Wilson, T. D. Science 317, 1351-1354 (2007).

11. Tversky, A. \& Kahneman, D. Cogn. Psychol. 5, 207-232 (1973).

12. Szpunar, K. K. \& Schacter, D. L. J. Exp. Psychol. Gen. 142, 323-327 (2013).
13. Bulley, A., Henry, J. D. \& Suddendorf, T. Conscious. Cogn. 49 53-69 (2017).

14. Scheier, M.F. \& Carver, C. S. Cognit. Ther. Res. 16, 201-228 (1992).

15. Karl, M., Kock, F., Ritchie, B. W. \& Gauss, J. Ann. Tour. Res. 87 103139 (2021).

Acknowledgements

A.B. is supported by an Australian National Health and Medical Research Council CJ Martin Biomedical Fellowship APP1162811 (GNT1162811), and an Australian Research Council Discovery Project Grant DP210101572. D.L.S. is supported by National Institute of Mental Health grant R01 MH060941 and National Institute on Aging grant R01 AG008441.

Competing interests

The authors declare no competing interests. 